\title{
Ehrhart clutters: Regularity and Max-Flow Min-Cut
}

\author{
José Martínez-Bernal \\ Departamento de Matemáticas \\ Centro de Investigación y de Estudios \\ Avanzados del IPN \\ Apartado Postal 14-740 \\ 07000 Mexico City, D.F. \\ jmb@math. cinvestav.mx
}

\author{
Edwin O'Shea* \\ Departamento de Matemáticas \\ Centro de Investigación y de Estudios \\ Avanzados del IPN \\ Apartado Postal 14-740 \\ 07000 Mexico City, D.F. \\ edwin@math.cinvestav.mx \\ Rafael H. Villarreal ${ }^{\dagger}$ \\ Departamento de Matemáticas \\ Centro de Investigación y de Estudios \\ Avanzados del IPN \\ Apartado Postal 14-740 \\ 07000 Mexico City, D.F. \\ vila@math.cinvestav.mx
}

Submitted: Mar 16, 2009; Accepted: Mar 15, 2010; Published: Mar 29, 2010

Mathematics Subject Classifications: 13H10, 52B20, 13D02, 90C47, 05C17, $05 \mathrm{C} 65$.

\begin{abstract}
If $\mathcal{C}$ is a clutter with $n$ vertices and $q$ edges whose clutter matrix has column vectors $\mathcal{A}=\left\{v_{1}, \ldots, v_{q}\right\}$, we call $\mathcal{C}$ an Ehrhart clutter if $\left\{\left(v_{1}, 1\right), \ldots,\left(v_{q}, 1\right)\right\} \subset\{0,1\}^{n+1}$ is a Hilbert basis. Letting $A(P)$ be the Ehrhart ring of $P=\operatorname{conv}(\mathcal{A})$, we are able to show that if $\mathcal{C}$ is a uniform unmixed MFMC clutter, then $\mathcal{C}$ is an Ehrhart clutter and in this case we provide sharp upper bounds on the Castelnuovo-Mumford regularity and the $a$-invariant of $A(P)$. Motivated by the Conforti-Cornuéjols conjecture on packing problems, we conjecture that if $\mathcal{C}$ is both ideal and the clique clutter of a perfect graph, then $\mathcal{C}$ has the MFMC property. We prove this conjecture for Meyniel graphs by showing that the clique clutters of Meyniel graphs are Ehrhart clutters. In much the same spirit, we provide a simple proof of our conjecture when $\mathcal{C}$ is a uniform clique clutter of a perfect graph. We close with a generalization of Ehrhart clutters as it relates to total dual integrality.
\end{abstract}

\footnotetext{
${ }^{*}$ Partially supported by SNI.

${ }^{\dagger}$ Partially supported by CONACyT grant 49251-F and SNI.
} 


\section{Introduction}

A clutter $\mathcal{C}$ is a family $E$ of subsets of a finite ground set $X$ such that if $S_{1}, S_{2} \in E$, then $S_{1} \not \subset S_{2}$. The ground set $X$ is called the vertex set of $\mathcal{C}$ and $E$ is called the edge set of $\mathcal{C}$, they are denoted by $V(\mathcal{C})$ and $E(\mathcal{C})$ respectively. Clutters are special hypergraphs and are sometimes called Sperner families in the literature. We can also think of a clutter as the maximal faces of a simplicial complex over a ground set. One example of a clutter is a graph with the vertices and edges defined in the usual way for graphs. For a thorough study of clutters and hypergraphs from the point of view of combinatorial optimization and commutative algebra see $[6,25]$ and $[11,14,16]$ respectively.

Let $\mathcal{C}$ be a clutter with vertex set $X=\left\{x_{1}, \ldots, x_{n}\right\}$ and with edge set $E(\mathcal{C})$. We shall assume that $\mathcal{C}$ has no isolated vertices, i.e., each vertex occurs in at least one edge and every edge contains at least two vertices. Permitting an abuse of notation, we will also denote by $x_{i}$ the $i^{\text {th }}$ variable in the polynomial ring $R=K\left[x_{1}, \ldots, x_{n}\right]$ over a field $K$. The edge ideal of $\mathcal{C}$, denoted by $I(\mathcal{C})$, is the monomial ideal of $R$ generated by all monomials

$x_{e}=\prod_{x_{i} \in e} x_{i}$ such that $e \in E(\mathcal{C})$. The assignment $\mathcal{C} \mapsto I(\mathcal{C})$ establishes a natural one to one correspondence between the family of clutters and the family of square-free monomial ideals. A subset $F$ of $X$ is called independent or stable if $e \not \subset F$ for any $e \in E(\mathcal{C})$. The dual concept of a stable vertex set is a vertex cover, i.e., a subset $C$ of $X$ is a vertex cover of $\mathcal{C}$ if and only if $X \backslash C$ is a stable vertex set. A first hint of the rich interaction between the combinatorics of $\mathcal{C}$ and the algebra of $I(\mathcal{C})$ is that the number of vertices in a minimum vertex cover of $\mathcal{C}$ (the covering number of $\mathcal{C}$ ) coincides with ht $I(\mathcal{C})$, the height of the ideal $I(\mathcal{C})$.

If $e$ is an edge of $\mathcal{C}$, its characteristic vector is the vector $v=\sum_{x_{i} \in e} e_{i}$, where $e_{i}$ is the $i^{\text {th }}$ unit vector in $\mathbb{R}^{n}$. Let $\mathcal{A}=\left\{v_{1}, \ldots, v_{q}\right\} \subset\{0,1\}^{n}$ denote the characteristic vectors of the edges of $\mathcal{C}$ and let $A$ denote the matrix whose columns, in order, are the vectors of $\mathcal{A}$. We call $A$ the clutter matrix or incidence matrix of $\mathcal{C}$. The Ehrhart ring of the lattice polytope $P=\operatorname{conv}(\mathcal{A})$ is the $K$-subring of $R[t]$ given by

$$
A(P)=K\left[\left\{x^{a} t^{b} \mid a \in b P \cap \mathbb{Z}^{n}\right\}\right],
$$

where $t$ is a new variable and $b P=\{b p \mid p \in P\}$ for each $b \in \mathbb{N}$. We use $x^{a}$ as an abbreviation for $x_{1}^{a_{1}} \cdots x_{n}^{a_{n}}$, where $a=\left(a_{i}\right) \in \mathbb{N}^{n}$. The homogeneous subring of $\mathcal{A}$ is the monomial subring

$$
K\left[x^{v_{1}} t, \ldots, x^{v_{q}} t\right] \subset R[t] .
$$

This ring is in fact a standard graded $K$-algebra because the vector $\left(v_{i}, 1\right)$ lies in the affine hyperplane with last coordinate equal to 1 for every $i$. In general we have the containment

$$
K\left[x^{v_{1}} t, \ldots, x^{v_{q}} t\right] \subset A(P),
$$

but as can be seen in $[9,14]$, the algebraic properties of edge ideals and Ehrhart rings of clutters are more tractable when the equality holds in this containment. We call such clutters Ehrhart clutters (or we say that the clutter is Ehrhart). 
A finite set $\mathcal{H} \subset \mathbb{Z}^{n}$ is called a Hilbert basis if $\mathbb{N} \mathcal{H}=\mathbb{R}_{+} \mathcal{H} \cap \mathbb{Z}^{n}$, where $\mathbb{R}_{+} \mathcal{H}$ and $\mathbb{N} \mathcal{H}$ are the non-negative real span and non-negative integer span respectively of $\mathcal{H}$. It is not hard to see that $\mathcal{C}$ is an Ehrhart clutter if and only if the $q$ vectors

$$
\left\{\left(v_{1}, 1\right), \ldots,\left(v_{q}, 1\right)\right\} \subset\{0,1\}^{n+1}
$$

form a Hilbert basis.

In this article we present two new families of Ehrhart clutters and we then use this information to study some algebraic properties of $I(\mathcal{C})$ and $A(P)$, such as normality, torsion freeness, Castelnuovo-Mumford regularity and $a$-invariant. The first two properties for edge ideals have already have been studied before in $[1,10,14,15,26]$. The CastelnuovoMumford regularity (see Definition 2.1) of a graded algebra is a numerical invariant that measures the "complexity" of its minimal graded free resolution and plays an important role in computational commutative algebra [3,22]. The $a$-invariant of the Ehrhart ring $A(P)$ is the largest integer $a \leqslant-1$ for which $-a P$ has an interior lattice point [2]. In Section 2 we introduce the regularity and the $a$-invariant in combinatorial and algebraic terms.

On the other hand, a clutter being Ehrhart will enable us to prove combinatorial properties, like when certain clutters have the max-flow min-cut property. This property is of central importance in combinatorial optimization [6] and so we define it here: the clutter $\mathcal{C}$ is said to have the max-flow min-cut property (or we say that $\mathcal{C}$ is MFMC) if the linear program:

$$
\max \{\langle\mathbf{1}, y\rangle \mid y \geqslant 0, A y \leqslant w\}
$$

has an integral optimal solution for all $w \in \mathbb{N}^{n}$. Here $\langle$,$\rangle denotes the standard inner$ product and $\mathbf{1}$ is the vector with all its entries equal to 1 .

The contents of this paper are as follows. The main theorem in Section 2 is a sharp upper bound for the Castelnuovo-Mumford regularity of $A(P)$. Before stating the theorem, recall that a clutter is called $d$-uniform if all its edges have size $d$. A clutter is called unmixed if all its minimal vertex covers have the same size. Unmixed clutters and $d$-uniform clutters have been studied in [23,32] and [8] respectively.

Theorem 2.3 If $\mathcal{C}$ is a d-uniform, unmixed $M F M C$ clutter with covering number $g$, then $\mathcal{C}$ is Ehrhart, the a-invariant of $A(P)$ is sharply bounded from above by $-g$, and the Castelnuovo-Mumford regularity of $A(P)$ is sharply bounded from above by $(d-1)(g-1)$.

A key ingredient to showing this result is a formula of Danilov-Stanley that expresses the canonical module of $A(P)$ using polyhedral geometry (see Eq. (2.5)). For uniform unmixed MFMC clutters, this formula can be made explicit enough (see Eq. (2.6)) to allow to prove our estimates for the regularity and the $a$-invariant of $A(P)$.

The blocker of a clutter $\mathcal{C}$, denoted by $\Upsilon(\mathcal{C})$, is the clutter whose edges are the minimal vertex covers of $\mathcal{C}$ (minimal with respect to inclusion). Sometimes the blocker of a clutter is referred to as the Alexander dual of the clutter. The edge ideal of $\Upsilon(\mathcal{C})$ is called the ideal of vertex covers of $\mathcal{C}$ or the Alexander dual of $I(\mathcal{C})$. As a corollary of Theorem 2.3, using the fact that the blocker of a bipartite graph satisfies the max-flow min-cut property [25], we obtain: 
Corollary 2.4 Let $G$ be an unmixed bipartite graph with $n$ vertices, let $\mathcal{A}=\left\{v_{1}, \ldots, v_{q}\right\}$ be the set of column vectors of the clutter matrix of the blocker of $G$, and let $P=\operatorname{conv}(\mathcal{A})$. Then the blocker of $G$ is Ehrhart and the Castelnuovo-Mumford regularity of $A(P)$ is bounded from above by $(n / 2)-1$.

In Section 3, we turn our attention to the clique clutters of Meyniel graphs. A clique of a graph is a set of mutually adjacent vertices. The clique clutter of a graph $G$, denoted by $\operatorname{cl}(G)$, is the clutter on $V(G)$ whose edges are the maximal cliques of $G$. The clutter matrix of $\operatorname{cl}(G)$ is called the vertex-clique matrix of $G$. A Meyniel graph is a simple graph in which every odd cycle of length at least five has at least two chords, where a chord of a cycle $C$ is an edge joining two non-adjacent vertices of $C$. A clutter $\mathcal{C}$ is called ideal if the polyhedron $Q(A)=\{x \mid x \geqslant 0 ; x A \geqslant 1\}$ has only integral vertices, where $A$ is the clutter matrix of $\mathcal{C}$. Our main result in Section 3 is:

Theorem 3.1 Let $\mathcal{C}$ be the clique clutter of a Meyniel graph. If $\mathcal{C}$ is ideal, then $\mathcal{C}$ is $M F M C$.

Central to proving this result is that the clique clutters of Meyniel graphs are Ehrhart, the proof of which arises chiefly from a polyhedral interpretation of a known characterization of Meyniel graphs (see Theorem 3.3) and the fact that the cone of a vertex over a graph preserves the Meyniel property (see Lemma 3.7). Theorem 3.1 can also be stated as follows: the clique clutter of a Meyniel graph $G$ is ideal if and only if $I^{i}=I^{(i)}$ for $i \geqslant 1$, where $I \subset R$ is the edge ideal of the clique clutter of $G$ and $I^{(i)}$ is the $i^{\text {th }}$ symbolic power of $I$. This algebraic perspective plays a starring role in the proof of Theorem 3.1 and will be described in great detail in Section 3.

Let us take this opportunity to justify the importance of Theorem 3.1. Inspired by Lovász's weak perfect graph theorem (see Theorem 3.4), Conforti and Cornuéjols conjectured [6, Conjecture 1.6] that if $\mathcal{C}$ has the packing property (i.e., the linear program (1.2) has an integer optimal solution for all $\left.\omega \in\{0,1, \infty\}^{n}\right)$, then $\mathcal{C}$ is also MFMC. However, the packing property has proved quite difficult to understand and so, given that the Edmonds-Giles theorem [24, Corollary 22.1c] implies that if $\mathcal{C}$ is MFMC then $\mathcal{C}$ is ideal, some energies have been devoted to instead asking: if $\mathcal{C}$ is an ideal clutter, then what additional properties on $\mathcal{C}$ will suffice for $\mathcal{C}$ to be MFMC? For example, one property that suffices is the diadic property [7, Theorem 1.3]. We conjecture that the following holds:

Conjecture 1.1 Let $\mathcal{C}$ be the clique clutter of a perfect graph. If $\mathcal{C}$ is ideal, then $\mathcal{C}$ is $M F M C$.

Experimentally, Conjecture 1.1 holds in each of the many distinct examples of perfect graphs in $[20, \S 7]$, verified using a combination of the computational programs Normaliz [4] and Polymake [13]. Since every Meyniel graph is perfect [25, Theorem 66.6], then Theorem 3.1 states that the conjecture holds for Meyniel graphs. Conjecture 1.1 also holds when the clique clutter $\mathcal{C}$ of a perfect graph is uniform [33, Corollary 2.9]. In Theorem 3.8 we provide a simpler alternative proof of the uniform case, again by showing that these clutters are Ehrhart. 
Section 3 is closed with two examples of clique clutters of perfect graphs. The first example shows that the common approach of Theorem 3.1 and Theorem 3.8 involving Ehrhart clutters is not one that can be relied upon to prove Conjecture 1.1 outright. The second example is a perfect graph whose clique clutter edge ideal is not normal, in sharp contrast to a central result of [33] which shows that the edge ideal of the blocker of a perfect graph is always normal. Thus finding a graph theoretical description for the normality of edge ideals of clique clutters of perfect graphs remains an open problem.

We close the paper by providing some characterizations of total dual integrality, using a generalization of Ehrhart clutters. We say that the system $x A \leqslant w$ is totally dual integral (TDI for short) if the minimum in the LP-duality equation

$$
\max \{\langle a, x\rangle \mid x A \leqslant w\}=\min \{\langle y, w\rangle \mid y \geqslant 0 ; A y=a\}
$$

has an integral optimum solution $y$ for each integral vector $a$ with finite minimum. Note that the MFMC property for a clutter $\mathcal{C}$ in the previous sections can be stated as $x\left[A \mid I_{n}\right] \leqslant$ $(-\mathbf{1} \mid 0)$ is TDI, where $A$ is the clutter matrix of $\mathcal{C}, \mathbf{1}$ is the vector of all 1 's and $I_{n}$ is an identity matrix.

A rational polyhedron $Q$ is called integral if $Q$ is the convex hull of the integral points in $Q$. A classical theorem of Edmonds and Giles is that if the system $x A \leqslant w$ is TDI, then the polyhedron $\{x \mid x A \leqslant w\}$ is integral [24, Corollary 22,1c]. Its converse does not hold in general so, similar to Section 3, it is natural to ask: what properties can be added to a matrix $A$ so that $\{x \mid x A \leqslant w\}$ being integral implies that $x A \leqslant w$ is TDI? For example, Lovász's weak perfect graph theorem mentioned above can be restated as such a converse holding. We show the following theorem:

Theorem 4.1 Let $A$ be an integral matrix with column vectors $v_{1}, \ldots, v_{q}$ and let $w=\left(w_{i}\right)$ be an integral vector. If the polyhedron $P=\{x \mid x A \leqslant w\}$ is integral and $\mathcal{H}(A, w)=$ $\left\{\left(v_{i}, w_{i}\right)\right\}_{i=1}^{q}$ is a Hilbert basis, then the system $x A \leqslant w$ is TDI.

Note that the set of vectors $\mathcal{H}(A, w)$ being a Hilbert basis is in some sense a generalization of Ehrhart clutters. We end the section with Proposition 4.2 describing a scenario where the converse to Theorem 4.1 holds.

\section{Castelnuovo-Mumford regularity and $a$-invariants}

We continue using the definitions and terms from the introduction. In this section we give sharp upper bounds for the regularity and the $a$-invariant of Ehrhart rings arising from uniform unmixed MFMC clutters.

First we introduce the $a$-invariant and the regularity in combinatorial and algebraic terms. Assume that $A(P)=K\left[x^{v_{1}} t, \ldots, x^{v_{q}} t\right]$, i.e., assume that $\mathcal{C}$ is an Ehrhart clutter. Then $A(P)$ becomes a standard graded $K$-algebra

$$
A(P)=\bigoplus_{i=0}^{\infty} A(P)_{i}
$$

THE ELECTRONIC JOURNAL OF COMBINATORICS 17 (2010), \#R52 
with $i^{\text {th }}$ component given by

$$
A(P)_{i}=\sum_{a \in \mathbb{Z}^{n} \cap i P} K x^{a} t^{i}
$$

A nice property of $A(P)$ is its normality, i.e., $A(P)$ is an integral domain which is integrally closed in its field of fractions [3, p. 276]. Therefore $A(P)$ is a Cohen-Macaulay domain by a theorem of Hochster [19]. The Hilbert series of $A(P)$ is given by

$$
F(A(P), z)=\sum_{i=0}^{\infty} \operatorname{dim}_{K} A(P)_{i} z^{i}=\sum_{i=0}^{\infty}\left|\mathbb{Z}^{n} \cap i P\right| z^{i},
$$

this series is called the Ehrhart series of $P$. By the Hilbert-Serre theorem [3, 27], and the fact that $A(P)$ is a Cohen-Macaulay domain, it follows that this is a rational function that can be uniquely written as:

$$
F(A(P), z)=\frac{h(z)}{(1-z)^{d+1}}=\frac{h_{0}+h_{1} z+\cdots+h_{s} z^{s}}{(1-z)^{d+1}}
$$

with $h(1)>0, h_{i} \in \mathbb{N}$ for all $i, h_{s}>0$ and $d=\operatorname{dim}(P)$. The a-invariant of $A(P)$, denoted by $a(A(P))$, is the degree of $F(A(P), z)$ as a rational function. This invariant is of combinatorial interest because it turns out that $-a(A(P))$ is the smallest integer $k \geqslant 1$ for which $k P$ has an interior lattice point (see [2, Theorem 6.51]).

The vector $h=\left(h_{0}, \ldots, h_{s}\right)$ is called the $h$-vector of $A(P)$. As $A(P)$ is a CohenMacaulay standard graded $K$-algebra, according to [30, Corollary B.4.1, p. 347], the number $s$ turns out to be $\operatorname{reg}(A(P)$ ), the Castelnuovo-Mumford regularity of $A(P)$ (see Definition 2.1). Thus $\operatorname{reg}(A(P))$ measures the size of the $h$-vector of $A(P)$ and we have the equality

$$
\operatorname{reg}(A(P))=\operatorname{dim}(A(P))+a(A(P)) .
$$

The $h$-vector of $A(P)$ is of interest in algebra and combinatorics [2, 3, 17, 22, 28] because it encodes information about the lattice polytope $P$ and the algebraic structure of $A(P)$. For instance $h(1)$ is the multiplicity of the ring $A(P)$ and $h(1)=d ! \operatorname{vol}(P)$, where $\operatorname{vol}(P)$ is the relative volume of $P$.

Next we give the definition of regularity of a homogeneous subring in terms of its minimal graded free resolution.

Definition 2.1 Let $S=K\left[x^{v_{1}} t, \ldots, x^{v_{q}} t\right]$ be a homogeneous subring with the standard grading induced by $\operatorname{deg}\left(x^{a} t^{b}\right)=b$. Let

$$
K\left[t_{1}, \ldots, t_{q}\right] / I_{\mathcal{A}} \simeq S, \quad \bar{t}_{i} \mapsto x^{v_{i}} t
$$

be a presentation of the homogeneous subring $S$, and let $\mathbb{F}_{\star}$ be the minimal graded resolution of $S$ by free $K\left[t_{1}, \ldots, t_{q}\right]$-modules. The Castelnuovo-Mumford regularity of $S$ is defined as $\operatorname{reg}(S)=\max \left\{b_{j}-j\right\}$, where $b_{j}$ is the maximum of the degrees of a minimal set of generators of $F_{j}$, the $j^{\text {th }}$ component of $\mathbb{F}_{\star}$. 
Proposition 2.2 [14, Proposition 5.8] Let $\mathcal{C}$ be a d-uniform clutter and let $A$ be its clutter matrix. If the polyhedron $Q(A)=\{x \mid x \geqslant 0 ; x A \geqslant 1\}$ is integral, then there are $X_{1}, \ldots, X_{d}$ mutually disjoint minimal vertex covers of $\mathcal{C}$ such that $X=\cup_{i=1}^{d} X_{i}$. In particular if $g_{1}, \ldots, g_{q}$ are the edges of $\mathcal{C},\left|X_{i} \cap g_{j}\right|=1$ for all $i, j$.

We come to the main result of this section.

Theorem 2.3 Let $\mathcal{C}$ be a d-uniform unmixed $M F M C$ clutter with covering number $g$ and let $\mathcal{A}=\left\{v_{1}, \ldots, v_{q}\right\}$ be the characteristic vectors of the edges of $\mathcal{C}$. If $A(P)$ is the Ehrhart ring of $P=\operatorname{conv}(\mathcal{A})$, then $\mathcal{C}$ is an Ehrhart clutter, the a-invariant of $A(P)$ is sharply bounded from above by $-g$ and the Castelnuovo-Mumford regularity of $A(P)$ is sharply bounded from above by $(d-1)(g-1)$.

Proof. Let $\mathcal{B}=\left\{\left(v_{i}, 1\right)\right\}_{i=1}^{q}$ and $\mathcal{A}^{\prime}=\mathcal{B} \cup\left\{e_{i}\right\}_{i=1}^{n}$, where $n$ is the number of vertices of $\mathcal{C}$ and $e_{i}$ is the $i^{\text {th }}$ unit vector. We first show the equality

$$
\mathbb{R}_{+} \mathcal{B}=\mathbb{R} \mathcal{B} \cap \mathbb{R}_{+} \mathcal{A}^{\prime}
$$

where $\mathbb{R} \mathcal{B}$ is the vector space spanned by $\mathcal{B}$ and $\mathbb{R}_{+} \mathcal{B}$ is the cone generated by $\mathcal{B}$. The left hand side is clearly contained in the right hand side. Conversely, take $(a, b)$ in the cone $\mathbb{R} \mathcal{B} \cap \mathbb{R}_{+} \mathcal{A}^{\prime}$, where $a \in \mathbb{R}^{n}$ and $b \in \mathbb{R}$. Then one has

$$
\begin{aligned}
& (a, b)=\eta_{1}\left(v_{1}, 1\right)+\cdots+\eta_{q}\left(v_{q}, 1\right) \quad\left(\eta_{i} \in \mathbb{R}\right), \\
& (a, b)=\lambda_{1}\left(v_{1}, 1\right)+\cdots+\lambda_{q}\left(v_{q}, 1\right)+\mu_{1} e_{1}+\cdots+\mu_{n} e_{n} \quad\left(\lambda_{i}, \mu_{j} \in \mathbb{R}_{+} \forall i, j\right) .
\end{aligned}
$$

For $a=\left(a_{i}\right) \in \mathbb{R}^{n}$, we set $|a|=\sum_{i} a_{i}$. Hence using that $\mathcal{C}$ is $d$-uniform, i.e., $\left|v_{i}\right|=d$ for all $i$, we get $b d=b d+\sum_{i} \mu_{i}$. This proves that $\mu_{i}=0$ for all $i$ and thus $(a, b)$ is in $\mathbb{R}_{+} \mathcal{B}$, as required.

Next we prove that $\mathcal{C}$ is an Ehrhart clutter, i.e., we will prove the equality

$$
K\left[x^{v_{1}} t, \ldots, x^{v_{q}} t\right]=A(P) .
$$

By [14, Theorem 4.6], the Rees algebra

$$
R[I(\mathcal{C}) t]=R\left[x^{v_{1}} t, \ldots, x^{v_{q}} t\right] \subset R[t]
$$

of the edge ideal $I(\mathcal{C})=\left(x^{v_{1}}, \ldots, x^{v_{q}}\right)$ is normal. Hence, using [9, Theorem 3.15], we obtain the required equality.

The next step in the proof is to find a good expression for the canonical module of $A(P)$ (see Eq. (2.6) below) that can be used to estimate the regularity and the $a$-invariant of $A(P)$. We begin by extracting some of the information encoded in the polyhedral representation of the cone $\mathbb{R}_{+} \mathcal{A}^{\prime}$. Let $C_{1}, \ldots, C_{s}$ be the minimal vertex covers of $\mathcal{C}$ and let $u_{k}=\sum_{x_{i} \in C_{k}} e_{i}$ for $1 \leqslant k \leqslant s$. By [14, Proposition 3.13 and Theorem 4.6] we obtain that the irreducible representation of $\mathbb{R}_{+} \mathcal{A}^{\prime}$ as an intersection of closed halfspaces is given by

$$
\mathbb{R}_{+} \mathcal{A}^{\prime}=H_{e_{1}}^{+} \cap \cdots \cap H_{e_{n+1}}^{+} \cap H_{\left(u_{1},-1\right)}^{+} \cap \cdots \cap H_{\left(u_{s},-1\right)}^{+} .
$$


Here $H_{a}^{+}$denotes the closed halfspace $H_{a}^{+}=\{x \mid\langle x, a\rangle \geqslant 0\}$ and $H_{a}$ stands for the hyperplane through the origin with normal vector $a$. Let $A$ be the clutter matrix of $\mathcal{C}$ whose columns are $v_{1}, \ldots, v_{q}$. The set covering polyhedron

$$
Q(A)=\{x \mid x \geqslant 0 ; x A \geqslant 1\}
$$

is integral [14, Theorem 4.6] and $\mathcal{C}$ is unmixed by hypothesis. Therefore, by Proposition 2.2, there are $X_{1}, \ldots, X_{d}$ mutually disjoint minimal vertex covers of $\mathcal{C}$ of size $g$ such that $X=\cup_{i=1}^{d} X_{i}$. Notice that $\left|X_{i} \cap f\right|=1$ for $1 \leqslant i \leqslant d$ and $f \in E(\mathcal{C})$. We may assume that $X_{i}=C_{i}$ for $1 \leqslant i \leqslant d$. Therefore, using Eqs. (2.1) and (2.3), we get

$$
\begin{aligned}
\mathbb{R}_{+} \mathcal{B} & =\mathbb{R} \mathcal{B} \cap \mathbb{R}_{+} \mathcal{A}^{\prime} \\
& =\mathbb{R} \mathcal{B} \cap H_{e_{1}}^{+} \cap \cdots \cap H_{e_{n+1}}^{+} \cap H_{\left(u_{1},-1\right)}^{+} \cap \cdots \cap H_{\left(u_{s},-1\right)}^{+} \\
& =\mathbb{R} \mathcal{B} \cap H_{e_{1}}^{+} \cap \cdots \cap H_{e_{n}}^{+} \cap H_{e_{n+1}}^{+} \cap\left(\cap_{i \in \mathcal{I}} H_{\left(u_{i},-1\right)}^{+}\right),
\end{aligned}
$$

where $i \in \mathcal{I}$ if and only if $H_{\left(u_{i},-1\right)}^{+}$defines a proper face of the cone $\mathbb{R}_{+} \mathcal{B}$. As $\left(v_{i}, 1\right)$ lies in the affine hyperplane $x_{n+1}=1$ for all $i$, the ring $A(P)$ becomes a graded $K$-algebra generated by monomials of degree 1 . Notice that a monomial $x^{a} t^{b}$ has degree $b$ in this grading. The Ehrhart ring $A(P)$ is a normal domain. Then, according to a well known formula of Danilov-Stanley [3, Theorem 6.3.5], its canonical module is the ideal of $A(P)$ given by

$$
\omega_{A(P)}=\left(\left\{x_{1}^{a_{1}} \cdots x_{n}^{a_{n}} t^{a_{n+1}} \mid a=\left(a_{i}\right) \in \mathbb{N} \mathcal{B} \cap\left(\mathbb{R}_{+} \mathcal{B}\right)^{\circ}\right\}\right),
$$

where $\left(\mathbb{R}_{+} \mathcal{B}\right)^{\circ}$ denotes the relative interior of the cone $\mathbb{R}_{+} \mathcal{B}$. Using Eqs. (2.2) and (2.4) we can express the canonical module as:

$$
\omega_{A(P)}=\left(\left\{x_{1}^{a_{1}} \cdots x_{n}^{a_{n}} t^{a_{n+1}} \mid\left(a_{i}\right) \in \mathbb{R} \mathcal{B} ; a_{i} \geqslant 1 \forall i ; \sum_{x_{i} \in C_{k}} a_{i} \geqslant a_{n+1}+1 \text { for } k \in \mathcal{I}\right\}\right) .
$$

Next we estimate the $a$-invariant of $A(P)$. Recall that the a-invariant of $A(P)$ is the degree, as a rational function, of the Hilbert series of $A(P)$ [31, p. 99]. The ring $A(P)$ is normal, then $A(P)$ is Cohen-Macaulay [19] and its $a$-invariant is given by

$$
a(A(P))=-\min \left\{i \mid\left(\omega_{A(P)}\right)_{i} \neq 0\right\},
$$

see [3, p. 141] and [31, Proposition 4.2.3]. Take an arbitrary monomial $x^{a} t^{b}=x_{1}^{a_{1}} \cdots x_{n}^{a_{n}} t^{b}$ in the ideal $\omega_{A(P)}$, with $b=a_{n+1}$. By Eqs. (2.4) and (2.6), the vector $(a, b)$ is in $\mathbb{R}_{+} \mathcal{B}$ and $a_{i} \geqslant 1$ for $i=1 \ldots, n$. Thus we can write

$$
(a, b)=\lambda_{1}\left(v_{1}, 1\right)+\cdots+\lambda_{q}\left(v_{q}, 1\right) \quad\left(\lambda_{i} \geqslant 0\right) .
$$

Since $\left\langle v_{i}, u_{k}\right\rangle=1$ for $i=1, \ldots, q$ and $k=1, \ldots, d$, we obtain

$$
g=\left|u_{k}\right| \leqslant \sum_{x_{i} \in C_{k}} a_{i}=\left\langle a, u_{k}\right\rangle=\lambda_{1}\left\langle v_{1}, u_{k}\right\rangle+\cdots+\lambda_{q}\left\langle v_{q}, u_{k}\right\rangle=\lambda_{1}+\cdots+\lambda_{q}=b
$$


for $1 \leqslant k \leqslant d$. This means that $\operatorname{deg}\left(x^{a} t^{b}\right) \geqslant g$. Consequently $-a(A(P)) \geqslant g$, as required. Next we show that $\operatorname{reg}(A(P)) \leqslant(d-1)(g-1)$. Since $A(P)$ is Cohen-Macaulay, we have

$$
\operatorname{reg}(A(P))=\operatorname{dim}(A(P))+a(A(P)) \leqslant \operatorname{dim}(A(P))-g,
$$

see [30, Corollary B.4.1, p. 347]. Using that $\left\langle v_{i}, u_{k}\right\rangle=1$ for $i=1, \ldots, q$ and $k=1, \ldots, d$, by induction on $d$ it is seen that $\operatorname{rank}(A) \leqslant g+(d-1)(g-1)$. Thus using the fact that $\operatorname{dim}(A(P))=\operatorname{rank}(A)$ and Eq. $(2.8)$, we get $\operatorname{reg}(A(P)) \leqslant(d-1)(g-1)$.

Finally, we now show that the upper bounds for the $a$-invariant and for the regularity are sharp. Let $\mathcal{C}$ be the clutter with vertex set $X=\cup_{i=1}^{d} X_{i}$ whose minimal vertex covers are exactly $X_{1}, \ldots, X_{d}$. Let $v_{1}, \ldots, v_{q}$ be the characteristic vectors of the edges of $\mathcal{C}$ and let $A$ be the matrix with column vectors $v_{1}, \ldots, v_{q}$. Using [25, Corollary 83.1a] (cf. [14, Corollary 4.26]) it is not hard to see that $\mathcal{C}$ satisfies the hypotheses of the theorem, i.e., the clutter $\mathcal{C}$ is MFMC, is $d$-uniform, unmixed and has covering number equal to $g$. Moreover the rank of $A$ is $g+(d-1)(g-1)$. Thus by Eq. (2.8) it suffices to show that $a(A(P))=-g$. Any edge of $\mathcal{C}$ intersects any minimal vertex cover of $\mathcal{C}$ in exactly one vertex. Therefore, using Eq. (2.4), we get

$$
\mathbb{R}_{+} \mathcal{B}=\mathbb{R} \mathcal{B} \cap H_{e_{1}}^{+} \cap \cdots \cap H_{e_{n}}^{+} \cap H_{e_{n+1}}^{+}
$$

Hence, using Eq. (2.6), we can express the canonical module as:

$$
\omega_{A(P)}=\left(\left\{x_{1}^{a_{1}} \cdots x_{n}^{a_{n}} t^{a_{n+1}} \mid a=\left(a_{i}\right) \in \mathbb{R} \mathcal{B} ; a_{i} \geqslant 1 \text { for } i=1, \ldots, n+1\right\}\right) .
$$

It is well known that MFMC clutters have the König property (i.e., the covering number equals the maximum number of mutually disjoint edges). Thus $\mathcal{C}$ has $g$ mutually disjoint edges whose union is $X$, by relabeling the $v_{i}$ 's if necessary, we may assume that $v_{1}, \ldots, v_{g}$ satisfy $1=v_{1}+\cdots+v_{g}$. Thus by Eq. (2.10), we get that the monomial $x_{1} \cdots x_{n} t^{g}$ belongs to $\omega_{A(P)}$. Consequently $a(A(P)) \geqslant-g$ and the equality $a(A(P))=-g$ follows.

Corollary 2.4 Let $G$ be an unmixed bipartite graph with $n$ vertices, let $\mathcal{A}=\left\{v_{1}, \ldots, v_{q}\right\}$ be the set of column vectors of the clutter matrix of the blocker of $G$, and let $P=\operatorname{conv}(\mathcal{A})$. Then the blocker of $G$ is an Ehrhart clutter and the Castelnuovo-Mumford regularity of $A(P)$ is sharply bounded from above by $(n / 2)-1$.

Proof. Let $\mathcal{C}=\Upsilon(G)$ be the clutter of minimal vertex covers of the bipartite graph $G$ and let $A$ be the matrix with column vectors $v_{1}, \ldots, v_{q}$. Since $A$ is the clutter matrix of $\mathcal{C}$ and all cycles of $G$ are even, it is well known [25, Theorem 83.1a(v)] that the clutter $\mathcal{C}$ has the max-flow min-cut property. The covering number of $\mathcal{C}$ is equal to 2 because the blocker of $\mathcal{C}$ is $G$. Moreover, as $G$ is bipartite and has no isolated vertices, it is seen that $n$ is even and that all edges of $\mathcal{C}$ have size $n / 2$ (see for instance [31, Lemma 6.4.2]). Therefore by Theorem 2.3, the Castelnuovo-Mumford regularity of $A(P)$ is bounded by $(n / 2)-1$. 


\section{Clique clutters with the Ehrhart Property}

The main result of this section is that Conjecture 1.1 holds for Meyniel graphs.

Theorem 3.1 Let $\mathcal{C}$ be the clique clutter of a Meyniel graph. If $\mathcal{C}$ is ideal, then $\mathcal{C}$ is $M F M C$.

We prove this result by studying the algebraic properties of edge ideals of clutters and by showing that clique clutters of Meyniel graphs are Ehrhart. As noted in the introduction, Conjecture 1.1 also holds for clique clutters of perfect graphs that are $d$-uniform (all edges have cardinality equal to $d$ ). We present a new simpler proof of that statement here, the heart of which is the same as the proof in the case of Meyniel graphs. Finally, we finish with examples of perfect graphs whose clique clutters are not Ehrhart, thus showing that a different approach than that presented here is needed to completely resolve Conjecture 1.1.

We begin with the necessary algebraic background. Let $\mathcal{C}$ be any clutter and let $C_{1}, \ldots, C_{s}$ be the minimal vertex covers of $\mathcal{C}$. By [31, Proposition 6.1.16], the primary decomposition of the edge ideal of $\mathcal{C}$ is given by

$$
I(\mathcal{C})=\mathfrak{p}_{1} \cap \cdots \cap \mathfrak{p}_{s}
$$

where $\mathfrak{p}_{i}=\left(C_{i}\right)$ for $1 \leqslant i \leqslant s$ and $\left(C_{i}\right)$ denotes the prime ideal of $R$ generated by the minimal vertex cover $C_{i}$. The $i^{\text {th }}$ symbolic power of $I=I(\mathcal{C})$ is the ideal of $R$ given by

$$
I^{(i)}=\mathfrak{p}_{1}^{i} \cap \cdots \cap \mathfrak{p}_{s}^{i},
$$

and the integral closure of $I^{i}$ is the ideal of $R$ given by (see [31]):

$$
\overline{I^{i}}=\left(\left\{x^{a} \in R \mid \exists p \geqslant 1 \text { such that }\left(x^{a}\right)^{p} \in I^{p i}\right\}\right) .
$$

A central result in this area shows that a clutter $\mathcal{C}$ is MFMC if and only if its edge ideal $I$ is normally torsion free, i.e., if and only if $I^{i}=I^{(i)}$ for $i \geqslant 1$ [15]. The proof of the following result is essentially the same as that made in [33, Corollary 2.9].

Theorem 3.2 Let $\mathcal{C}$ be a clutter. If $\mathcal{C}$ is both Ehrhart and ideal, then $\mathcal{C}$ is $M F M C$.

Proof. Let $\left\{v_{1}, \ldots, v_{q}\right\}$ be the set of columns of the clutter matrix of $\mathcal{C}$ and let $I=I(\mathcal{C})$ be the edge ideal of $\mathcal{C}$. Assuming that $\mathcal{C}$ is an Ehrhart clutter, we show that the following four conditions are equivalent:

(i) $\mathcal{C}$ is MFMC.

(ii) $I^{i}=I^{(i)}$ for $i \geqslant 1$.

(iii) $\overline{I^{i}}=I^{(i)}$ for $i \geqslant 1$.

(iv) $\mathcal{C}$ is ideal. 
(i) $\Rightarrow$ (ii): This was shown in [15, Corollary 3.14]. (ii) $\Rightarrow$ (iii): Follows readily because in general one has the inclusions $I^{i} \subset \overline{I^{i}} \subset I^{(i)}$ for $i \geqslant 1$. (iii) $\Rightarrow$ (iv): This was shown in [15, Corollary 3.13]. (iv) $\Rightarrow$ (i): By our hypothesis $\mathcal{C}$ is Ehrhart, which is equivalent to saying that $K\left[x^{v_{1}} t, \ldots, x^{v_{q}} t\right]=A(P)$, where $P=\operatorname{conv}\left(v_{1}, \ldots, v_{q}\right)$. As $\mathcal{C}$ is ideal, a direct application of [14, Proposition 4.4 and Theorem 4.6] gives that the clutter $\mathcal{C}$ is MFMC. Thus the four conditions are equivalent. Therefore if $\mathcal{C}$ is Ehrhart and ideal, then $\mathcal{C}$ is MFMC.

To prove Theorem 3.1 we will show that the clique clutters of Meyniel graphs are Ehrhart and then apply Theorem 3.2. A starting point to proving Conjecture 1.1 for Meyniel graphs is the following classification:

Theorem 3.3 (Hoáng [18]) A graph $G$ is Meyniel if and only if for each induced subgraph $H$ and for each vertex $u$ of $H$, there exists a stable set in $H$ that contains $u$ and this set intersects all maximal cliques of $H$.

Using this classification we will show that if $\mathcal{C}$ is the clique clutter of a Meyniel graph such that $\left\{v_{1}, \ldots, v_{q}\right\}$ are the characteristic vectors of the edges of $\mathcal{C}$, then $\left\{\left(v_{1}, 1\right), \ldots,\left(v_{q}, 1\right)\right\}$ is a Hilbert basis. By Theorem 3.2 we will then have a proof of Theorem 3.1.

We begin by recalling the polyhedral weak perfect graph theorem. Here the complement of a graph $G$ on the vertex set $\left\{x_{1}, \ldots, x_{n}\right\}$ is the graph $G^{c}$ on the same vertices as $G$ but whose maximal cliques are precisely the maximal stable sets of $G$.

Theorem 3.4 (Lovász [21], Chvátal [5], Fulkerson [12]) A graph G is perfect if and only if its complement is perfect if and only if the stability polytope $\operatorname{Stab}(G):=\{x \mid x \geqslant 0 ; x A \leqslant \mathbf{1}\}$ is integral if and only if the system $x \geqslant 0 ; x A \leqslant \mathbf{1}$ is TDI, where $A$ is the vertex-clique matrix of the graph $G$.

In this case the vertices of the stability polytope of $G$ are precisely the characteristic vectors of the stable sets of $G$. Finally, a theorem tying TDI with Hilbert bases: without loss of generality, every system of inequalities can be rewritten in the form $x A \leqslant w$. If $F$ is a face of the polyhedron $\{x \mid x A \leqslant w\}$ we say that a column of $A$ is active in $F$ if the corresponding inequality in $x A \leqslant w$ is satisfied with equality for all vectors in $F$.

Theorem 3.5 [24, Theorem 22.5] The system $x A \leqslant w$ is TDI if and only if for each face $F$ of the polyhedron $P=\{x \mid x A \leqslant w\}$, the columns of $A$ which are active in $F$ form a Hilbert basis.

By [25, Theorem 66.6], every Meyniel graph is perfect and so we can put Theorem 3.4 and Theorem 3.5 to good use for Meyniel graphs.

Lemma 3.6 Let $G$ be a Meyniel graph and let $\mathcal{A}=\left\{v_{1}, \ldots, v_{q}\right\}$ be the set of columns of the vertex-clique matrix of $G$. Then $\mathcal{A}=\left\{v_{1}, \ldots, v_{q}\right\}$ is a Hilbert basis. 
Proof. By Theorem 3.3, for each $x_{k}$ in $V(G)=\left\{x_{1}, \ldots, x_{n}\right\}$ there exists a stable set $B_{k}$ of $G$ containing $x_{k}$ and intersecting all maximal cliques of $G$. Let $\beta_{k}=\sum_{x_{i} \in B_{k}} e_{i}$ be the characteristic vector of $B_{k}$ for $1 \leqslant k \leqslant n$. Note that in general a clique of $G$ and a stable set of $G$ can meet in at most one vertex. Then for each $k=1,2, \ldots, n$ we have $\left\langle e_{k}, \beta_{k}\right\rangle=1$ and $\left\langle v_{j}, \beta_{k}\right\rangle=1$ for all $j$. Next, let $\beta:=\frac{1}{n} \sum_{k=1}^{n} \beta_{k}$. Note that $\beta$ also has the property that

$$
\left\langle v_{j}, \beta\right\rangle=1 \text { and }\left\langle e_{i}, \beta\right\rangle>0, \quad \forall i, j .
$$

Hence, $\beta$ is in the common intersection of all faces of $\operatorname{stab}(G)$ of the form $\left\langle v_{j}, x\right\rangle=1$. Call this intersection $F_{\beta}$. Thus $\beta$ belongs to the face $F_{\beta}$ of the stability polytope of $G$, rewritten as $\left\{x: x\left[A \mid-I_{n}\right] \leqslant(\mathbf{1} \mid 0)\right\}$, where $I_{n}$ is the $n \times n$ identity matrix and $(\mathbf{1} \mid 0)$ is the vector with $q 1$ 's followed by $n$ 0's. By [25, Theorem 66.6], every Meyniel graph is perfect and so by Theorem 3.4 the system $x\left[A \mid-I_{n}\right] \leqslant(\mathbf{1}, 0)$ is TDI. Now by Eq. (3.1), the columns of $\left[A \mid-I_{n}\right]$ which are active in $F_{\beta}$ are precisely the columns of $A$. By Theorem 3.5 , the columns of $\left[A \mid-I_{n}\right]$ which are active in $F_{\beta}$ form a Hilbert basis, i.e., $\left\{v_{1}, \ldots, v_{q}\right\}$ is a Hilbert basis as claimed.

Next, the suspension of a clutter $\mathcal{C}$ is the clutter $\mathcal{C}^{+}$with vertex set $X \cup\left\{x_{n+1}\right\}$ and edge set $\left\{e \cup\left\{x_{n+1}\right\} \mid e \in E(\mathcal{C})\right\}$, where $X=\left\{x_{1}, \ldots, x_{n}\right\}$ is the vertex set of $\mathcal{C}$. The cone $C(G)$, over a graph $G$, is the graph obtained by adding a new vertex $x_{n+1}$ to $G$ and joining every vertex of $G$ to $x_{n+1}$. If $G$ is a graph and $\operatorname{cl}(G)$ is its clique clutter, these two construction are related by $\operatorname{cl}(C(G))=\operatorname{cl}(G)^{+}$. The following is a simple lemma showing that the cone operation preserves both Meyniel-ness and perfection in graphs, and the suspension operation preserves idealness in a clutter.

Lemma 3.7 Let $G$ be a graph and let $\mathcal{C}$ be a clutter on the vertex set $X$. Then: (i) $G$ is Meyniel if and only if $C(G)$ is Meyniel. (ii) $G$ is perfect if and only if $C(G)$ is perfect. (iii) $\mathcal{C}$ is ideal if and only if $\mathcal{C}^{+}$is ideal.

Proof. (i) This follows immediately from the classification for Meyniel graphs in Theorem 3.3 and the construction of $C(G)$.

(ii) This follows from the complement of the cone over $G, C(G)^{c}$ equals the complement of $G$ with the isolated vertex $\left\{x_{n+1}\right\}$ and from a graph being perfect if and only if its complement is perfect (see Theorem 3.4).

(iii) This requires a little more work but the proof is still straightforward. Lehman [6, Theorem 1.17] showed that a clutter is ideal if and only if its blocker (defined in the introduction) is ideal. We now show that the clutter $\mathcal{C}$ is ideal if and only if $\mathcal{C}^{+}$is ideal.

Consider the blocker $\Upsilon\left(\mathcal{C}^{+}\right)$of the clutter $\mathcal{C}^{+}$. The edges of $\Upsilon\left(\mathcal{C}^{+}\right)$are precisely the edges of $\Upsilon(\mathcal{C})$ with the solitary edge $\left\{x_{n+1}\right\}$ (viewing the vertex as an edge). Let $B$ (resp. $\left.B^{+}\right)$denote the clutter matrix for the blocker of $\mathcal{C}\left(\right.$ resp. $\left.\mathcal{C}^{+}\right)$. By the decomposition theorem for polyhedra [24, 88.9$]$, the polyhedron $Q\left(B^{+}\right)=\left\{x \mid x \geqslant 0 ; x B^{+} \geqslant 1\right\}$ will simply be

$$
\left(\operatorname{conv}\left(\left(w_{1}, 0\right), \ldots,\left(w_{t}, 0\right)\right)+\mathbb{R}_{+}^{n+1}\right) \cap\left\{y \in \mathbb{R}_{+}^{n+1}: y_{n+1} \geqslant 1\right\},
$$

where $w_{1}, \ldots, w_{t} \in \mathbb{R}^{n}$ are the vertices of $Q(B)$. But the intersection above is simply

$$
\operatorname{conv}\left(\left(w_{1}, 1\right), \ldots,\left(w_{t}, 1\right)\right)+\mathbb{R}_{+}^{n+1} .
$$


Hence with this formulation we see that $\mathcal{C}$ is ideal if and only if $\Upsilon(\mathcal{C})$ is ideal if and only if $w_{1}, \ldots, w_{t} \in \mathbb{R}^{n}$ are integral if and only if $\left(w_{1}, 1\right), \ldots,\left(w_{t}, 1\right)$ are integral if and only if $\Upsilon\left(\mathcal{C}^{+}\right)$is ideal if and only if $\mathcal{C}^{+}$is ideal.

Note that if $\left\{v_{1}, \ldots, v_{q}\right\} \subset\{0,1\}^{n}$ are the characteristic vectors of the edges of the clutter $\mathcal{C}$, then $\left\{\left(v_{1}, 1\right), \ldots,\left(v_{q}, 1\right)\right\} \subset\{0,1\}^{n+1}$ are the characteristic vectors of the edges of the clutter $\mathcal{C}^{+}$. With Lemma 3.7(i) we are now ready prove our main result:

Proof of Theorem 3.1: If $G$ is a Meyniel graph, then so is $C(G)$, the cone over the graph $G$. The clutter matrix of $\operatorname{cl}(G)^{+}$is the matrix $A^{+}$whose columns are precisely $\left\{\left(v_{1}, 1\right), \ldots,\left(v_{q}, 1\right)\right\}$. Now applying Lemma 3.6 to the Meyniel graph $C(G)$ and noticing the equality $\operatorname{cl}(C(G))=\operatorname{cl}(G)^{+}$, we get that $\left\{\left(v_{1}, 1\right), \ldots,\left(v_{q}, 1\right)\right\}$ is a Hilbert basis, i.e., $\operatorname{cl}(G)$ is an Ehrahrt clutter. In addition, if we assume that the clique clutter of $G$ is ideal, then by Theorem 3.2 the clique clutter must also be MFMC.

Using arguments very similar to the above for Meyniel graphs, we now give a simpler proof of Conjecture 1.1 for uniform clutters which was first proved in [33, Corollary 2.9].

Theorem 3.8 Let $\mathcal{C}$ be the clique clutter of a perfect graph with edges $\left\{v_{1}, \ldots, v_{q}\right\}$ that is both ideal and uniform. Then (a) $\left\{v_{1}, \ldots, v_{q}\right\}$ is a Hilbert basis. (b) $\mathcal{C}$ is Ehrhart. (c) $\mathcal{C}$ is $M F M C$.

Proof. Recall that a clutter $\mathcal{C}$ is $d$-uniform if all edges have the same cardinality $d$. Let $g_{1}, \ldots, g_{q}$ be the edges of $\mathcal{C}$. From Proposition 2.2 we have that if $\mathcal{C}$ is ideal, then there are mutually disjoint sets $X_{1}, \ldots, X_{d}$ whose union is $X$ and such that $\left|g_{j} \cap X_{i}\right|=1$ for each $j=1, \ldots, q$ and each $i=1, \ldots, d$. A simple proof of this can be seen in [8, Proposition 2.2]. If $\mathcal{C}$ is also the clique clutter of a perfect graph $G$, then each of the $X_{i}$ 's are also maximal stable sets for the graph $G$.

(a) Let $\chi_{i}$ represent the $\{0,1\}$-characteristic vector of $X_{i}$ for every $i=1, \ldots, d$ and

$$
\gamma:=\frac{1}{d}\left(\chi_{1}+\cdots+\chi_{d}\right)=\left(\frac{1}{d}, \ldots, \frac{1}{d}\right) .
$$

Just as in the case of the constructed $\beta$ for Meyniel graphs, $\gamma$ also has the similar property that

$$
\left\langle v_{j}, \gamma\right\rangle=1 \text { and }\left\langle e_{i}, \gamma\right\rangle=1 / d>0, \quad \forall i, j,
$$

and, by the exact same argument as that for $\beta$ in Lemma 3.6, $\gamma$ belongs to a face $F_{\gamma}$ of the stability polytope of $G$, and the columns of $\left[A \mid-I_{n}\right]$ that are active in $F_{\gamma}$ are precisely the columns of $A$ and they form a Hilbert basis.

(b) By Lemma 3.7 (parts (ii) and (iii)), cones preserve perfection and suspensions preserve idealness respectively. Since $\mathcal{C}=\operatorname{cl}(G)$ is $d$-uniform, then $\mathcal{C}^{+}=\operatorname{cl}(C(G))$ is $(d+1)$-uniform. Hence part (a) can be applied to the clutter $\mathcal{C}^{+}$and so $\left\{\left(v_{1}, 1\right), \ldots,\left(v_{q}, 1\right)\right\}$ forms a Hilbert basis.

(c) Given part (b), its a simple consequence of Theorem 3.2. 
Remark 3.9 The clutter $\mathcal{C}$ being Ehrhart is central to both arguments and so classifying other clique clutters that are Ehrhart is certainly of interest. The clique clutter of the perfect graph in Example 3.10 below is not Ehrhart yet it is ideal and MFMC (confirmed computationally). Hence, to completely resolve Conjecture 1.1 an approach that differs from the one presented here is needed.

Example 3.10 Let $\mathcal{K}_{2,4}$ be the complete bipartite graph with vertex set $X=V_{1} \cup V_{2}$ and bipartition $V_{1}=\left\{x_{1}, x_{2}\right\}, V_{2}=\left\{x_{3}, x_{4}, x_{5}, x_{6}\right\}$. The matrix

$$
\left(\begin{array}{llllllll}
1 & 1 & 1 & 1 & 0 & 0 & 0 & 0 \\
0 & 0 & 0 & 0 & 1 & 1 & 1 & 1 \\
1 & 0 & 0 & 0 & 1 & 0 & 0 & 0 \\
0 & 1 & 0 & 0 & 0 & 1 & 0 & 0 \\
0 & 0 & 1 & 0 & 0 & 0 & 1 & 0 \\
0 & 0 & 0 & 1 & 0 & 0 & 0 & 1
\end{array}\right)
$$

is the incidence matrix of $\mathcal{K}_{2,4}$. Thus this matrix is totally unimodular and its rows are the maximal cliques of some perfect graph $G$ [25, Theorem 82.4], actually $G$ is the line graph of the bipartite graph $\mathcal{K}_{2,4}$ :

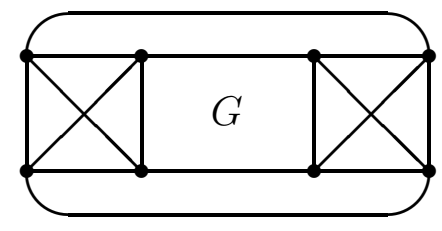

Using Normaliz [4] it is seen that $K\left[x^{v_{1}} t, \ldots, x^{v_{6}} t\right] \subsetneq A(P)$, where $v_{1}, \ldots, v_{6}$ are the rows of the matrix $B$. The extra element in $A(P)$ but not in $K\left[x^{v_{1}} t, \ldots, x^{v_{6}} t\right]$ is the monomial with exponent vector $(1,1,1,1,1,1,3)$.

The next example was constructed so that the clique clutter of the graph $G$ has a minor (in the sense of hypergraph theory [6]) whose edge ideal is not normal.

Example 3.11 Consider the following graph $G$ with 13 vertices:

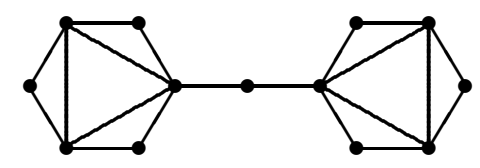

The ideal edge $I=I(\operatorname{cl}(G))$ of the clique clutter of $G$ is not normal. This graph is chordal, hence perfect. Thus edge ideals of clique clutters of perfect graphs are in general not normal. 


\section{TDI systems}

As already noted in the introduction, the MFMC property for a clutter $\mathcal{C}$ can be stated as $x\left[A \mid I_{n}\right] \leqslant(-\mathbf{1} \mid 0)$ is TDI, where $A$ is the clutter matrix of $\mathcal{C}$. Thus we have already seen (a small slice of) the central role that TDI plays in combinatorial optimization. Another motivation to study TDI systems is, for cost vectors $w \in \mathbb{N}^{q}$ for which the problem $\min \{\langle y, w\rangle \mid y \geqslant 0 ; A y=a\}$ has a unique solution, the system $x A \leqslant w$ is TDI if and only if the toric initial ideal of $A$ with respect to $w$ is generated by square-free monomials [29, Corollary. 8.9].

In the previous sections we saw how Ehrhart clutters facilitated results on the regularity of edge ideals and the MFMC property of clutters. In this short closing section we replace the $\{0,1\}$-clutter matrix with a general integer matrix $A$ whose columns, as before, we denote by the set $\left\{v_{1}, \ldots, v_{q}\right\}$ and we replace the vector 1 of all ones of length $q$ with a general vector $w=\left(w_{1}, \ldots, w_{q}\right) \in \mathbb{Z}^{q}$. Motivated by our results for Ehrhart clutters we can ask: is there any significance to $\mathcal{H}(A, w):=\left\{\left(v_{1}, w_{1}\right), \ldots,\left(v_{q}, w_{q}\right)\right\}$ being a Hilbert basis, especially with regards to the TDI property?

Theorem 4.1 Let $A$ be an integral matrix with column vectors $v_{1}, \ldots, v_{q}$ and let $w=\left(w_{i}\right)$ be an integral vector. If the polyhedron $P=\{x \mid x A \leqslant w\}$ is integral and $\mathcal{H}(A, w)=$ $\left\{\left(v_{i}, w_{i}\right)\right\}_{i=1}^{q}$ is a Hilbert basis, then the system $x A \leqslant w$ is TDI.

Proof. To show that $x A \leqslant w$ is TDI it suffices to show that the second part of the equivalence in Theorem 3.5 holds for the minimal faces of $P$. Let $F$ be such a minimal face of $P$. We may assume that $v_{1}, \ldots, v_{r}$ are the columns of $A$ which are active in $F$. Then $\left\langle x, v_{i}\right\rangle=w_{i}$ for $x \in F$ and $1 \leqslant i \leqslant r$. If $\left\langle y, v_{i}\right\rangle<w_{i}$ for some $y \in F$, then $\left\langle x, v_{i}\right\rangle<w_{i}$ for any other $x \in F$. Indeed if $\left\langle x, v_{i}\right\rangle=w_{i}$ for some $x \in F$, consider the supporting hyperplane of $P$ given by $H=\left\{x \mid\left\langle x, v_{i}\right\rangle=w_{i}\right\}$, then $x \in F \cap H \subsetneq F$ because $y \in F$ and $y \notin F \cap H$, a contradiction to the minimality of the face $F$. Thus we may also assume that $\left\langle x, v_{i}\right\rangle<w_{i}$ for $x \in F$ and $i>r$. Since $P$ is integral, each face of $P$ contains integral vectors, see [24, Section 16.3]. Pick an integral vector $x_{0} \in F$. We can now show that $\mathcal{B}=\left\{v_{1}, \ldots, v_{r}\right\}$ is a Hilbert basis.

Let $a \in \mathbb{R}_{+} \mathcal{B} \cap \mathbb{Z}^{n}$. Then we can write $a=\lambda_{1} v_{1}+\cdots+\lambda_{r} v_{r}$ where $\lambda_{i} \geqslant 0$ for each $i=1, \ldots, r$. Thus we have

$$
b:=\left\langle a, x_{0}\right\rangle=\lambda_{1}\left\langle v_{1}, x_{0}\right\rangle+\cdots+\lambda_{r}\left\langle v_{r}, x_{0}\right\rangle=\lambda_{1} w_{1}+\cdots+\lambda_{r} w_{r} .
$$

In particular $b$ is an integer and we can write $(a, b)=\lambda_{1}\left(v_{1}, w_{1}\right)+\cdots+\lambda_{r}\left(v_{r}, w_{r}\right)$. By hypothesis $\mathcal{H}(A, w)$ is a Hilbert basis. Therefore we can write $(a, b)=\eta_{1}\left(v_{1}, w_{1}\right)+\cdots+$ $\eta_{q}\left(v_{q}, w_{q}\right)$ where $\eta_{i} \in \mathbb{N}$ for each $i=1, \ldots, q$. Therefore

$$
0=\left\langle(a, b),\left(x_{0},-1\right)\right\rangle=\sum_{i=1}^{r} \eta_{i} \underbrace{\left\langle\left(v_{i}, w_{i}\right),\left(x_{0},-1\right)\right\rangle}_{=0}+\sum_{i=r+1}^{q} \eta_{i} \underbrace{\left\langle\left(v_{i}, w_{i}\right),\left(x_{0},-1\right)\right\rangle}_{<0} .
$$

Hence $\eta_{i}=0$ for $i>r$ and $a=\eta_{1} v_{1}+\cdots+\eta_{r} v_{r}$. Thus $a \in \mathbb{N} \mathcal{B}$, as required. 
One motivation for the above theorem is [24, Theorem 22.18], which states that the system $x A \leqslant w$ has the integer rounding property if and only if the set

$$
\left\{\left(v_{1}, w_{1}\right), \ldots,\left(v_{q}, w_{q}\right), e_{n+1}\right\} \subset \mathbb{Z}^{n+1}
$$

is a Hilbert basis. A straightforward corollary of this result is that we have an "if and only if" in Theorem 4.1 if we append the vector $e_{n+1}$ onto $\mathcal{H}(A, w)$. The converse of Theorem 4.1 is not true in general but there are interesting systems for which the converse does hold. Let $A$ be an integral matrix and let $w$ be an integral vector. As before, we can rewrite the system $x \geqslant 0 ; x A \leqslant w$ as $x\left[A \mid-I_{n}\right] \leqslant(w \mid 0)$.

Proposition 4.2 Let $A$ be a non-negative integral matrix of order $n \times q$ with column vectors $v_{1}, \ldots, v_{q}$ and let $w=\left(w_{i}\right) \in \mathbb{N}^{q}$. Then the system $x \geqslant 0 ; x A \leqslant w$ is TDI if and only if the polyhedron $P=\{x \mid x \geqslant 0 ; x A \leqslant w\}$ is integral and

$$
\mathcal{H}:=\mathcal{H}\left(\left[A \mid-I_{n}\right],(w \mid 0)\right)=\left\{\left(v_{1}, w_{1}\right), \ldots,\left(v_{q}, w_{q}\right),-e_{1}, \ldots,-e_{n}\right\}
$$

is a Hilbert basis.

Proof. The "if" part follows from Theorem 4.1. For the "only if" part, assume that the system $x \geqslant 0 ; x A \leqslant w$ is TDI. By the Edmonds-Giles theorem [24, Corollary 22.1c], the polyhedrom $P$ must be integral. All that remains to show is that $\mathcal{H}$ is a Hilbert basis. Take $(a, b) \in \mathbb{R}_{+} \mathcal{H} \cap \mathbb{Z}^{n+1}$, where $a \in \mathbb{Z}^{n}$ and $b \in \mathbb{Z}$. By hypothesis, the linear program $\min \{\langle y, w\rangle \mid y \geqslant 0 ; A y \geqslant a\}$ has an integral optimum solution $y=\left(y_{i}\right)$ such that $\langle y, w\rangle \leqslant b$. Since $y \geqslant 0$ and $a \leqslant A y$, we can write $a=y_{1} v_{1}+\cdots+y_{q} v_{q}-\delta_{1} e_{1}-\cdots-\delta_{n} e_{n}$ where each $\delta_{i} \in \mathbb{N}$. This implies that

$(a, b)=y_{1}\left(v_{1}, w_{1}\right)+\cdots+y_{q-1}\left(v_{q-1}, w_{q-1}\right)+\left(y_{q}+b-\langle y, w\rangle\right)\left(v_{q}, w_{q}\right)-(b-\langle y, w\rangle) v_{q}-\delta$,

where $\delta=\left(\delta_{i}\right)$. As the entries of $A$ are in $\mathbb{N}$, the vector $-v_{q}$ can be written as a nonnegative integer combination of $-e_{1}, \ldots,-e_{n}$. Thus $(a, b) \in \mathbb{N} \mathcal{H}$ as claimed.

\section{References}

[1] J. P. Brennan, L. A. Dupont and R. H. Villarreal, Duality, a-invariants and canonical modules of rings arising from linear optimization problems, Bull. Math. Soc. Sci. Math. Roumanie (N.S.) 51 (2008), no. 4, 279-305.

[2] W. Bruns and J. Gubeladze, Polytopes, rings, and K-theory, Springer Monographs in Mathematics, Springer, 2009.

[3] W. Bruns and J. Herzog, Cohen-Macaulay Rings, Cambridge University Press, Cambridge, Revised Edition, 1997.

[4] W. Bruns and B. Ichim, Normaliz 2.0, Computing normalizations of affine semigroups 2008. Available from

http://www.math.uos.de/normaliz. 
[5] V. Chvátal, On certain polytopes associated with graphs, J. Combin. Theory Ser. B 18 (1975), 138-154.

[6] G. Cornuéjols, Combinatorial optimization: Packing and covering, CBMS-NSF Regional Conference Series in Applied Mathematics 74, SIAM (2001).

[7] G. Cornuéjols, B. Guenin and F. Margot, The Packing Property, Math. Programming 89 (2000), 113-126.

[8] L. A. Dupont and R. H. Villarreal, Algebraic and combinatorial properties of ideals and algebras of uniform clutters of TDI systems, J. Comb. Optim., to appear.

[9] C. Escobar, J. Martínez-Bernal and R. H. Villarreal, Relative volumes and minors in monomial subrings, Linear Algebra Appl. 374 (2003), 275-290.

[10] C. Escobar, R. H. Villarreal and Y. Yoshino, Torsion freeness and normality of blowup rings of monomial ideals, in Commutative Algebra: Geometric, Homological, Combinatorial and Computational Aspects, Proceedings: Sevilla and Lisbon (A. Corso et al., Eds.), Lect. Notes Pure Appl. Math. 244, Chapman \& Hall/CRC, Boca Raton, FL, 2006, pp. 69-84.

[11] S. Faridi, Cohen-Macaulay properties of square-free monomial ideals, J. Combin. Theory Ser. A 109(2) (2005), 299-329.

[12] D. R. Fulkerson, Anti-blocking polyhedra, J. Combin. Theory Ser. B 12 (1972), 5071.

[13] E. Gawrilow, M. Joswig, Polymake: a Framework for Analyzing Convex Polytopes, 43-74, in Polytopes, Combinatorics and Computation, Birkhäuser, 2000.

[14] I. Gitler, E. Reyes and R. H. Villarreal, Blowup algebras of square-free monomial ideals and some links to combinatorial optimization problems, Rocky Mountain J. Math. 39 (2009), no. 1, 71-102.

[15] I. Gitler, C. Valencia and R. H. Villarreal, A note on Rees algebras and the MFMC property, Beiträge Algebra Geom. 48 (2007), no. 1, 141-150.

[16] H. T. Hà and A. Van Tuyl, Monomial ideals, edge ideals of hypergraphs, and their graded Betti numbers, J. Algebraic Combin. 27 (2008), 215-245.

[17] T. Hibi, Ehrhart polynomials of convex polytopes, $h$-vectors of simplicial complexes, and nonsingular projective toric varieties, Discrete and computational geometry (New Brunswick, NJ, 1989/1990), 165-177, DIMACS Ser. Discrete Math. Theoret. Comput. Sci., 6, Amer. Math. Soc., Providence, RI, 1991.

[18] C. T. Hoáng, On a conjecture of Meyniel, J. Combin. Theory Ser. B 42 (1987), no. $3,302-312$.

[19] M. Hochster, Rings of invariants of tori, Cohen-Macaulay rings generated by monomials, and polytopes, Ann. of Math. 96 (1972), 318-337.

[20] S. Hougardy, Classes of perfect graphs, Discrete Math. 306 (2006), 2529-2571.

[21] L. Lovász, Normal hypergraphs and the perfect graph conjecture, Discrete Math. 2 (1972), 253-267. 
[22] E. Miller and B. Sturmfels, Combinatorial Commutative Algebra, Graduate Texts in Mathematics 227, Springer, 2004.

[23] S. Morey, E. Reyes and R. H. Villarreal, Cohen-Macaulay, shellable and unmixed clutters with a perfect matching of König type, J. Pure Appl. Algebra 212 (2008), no. $7,1770-1786$.

[24] A. Schrijver, Theory of Linear and Integer Programming, John Wiley \& Sons, New York, 1986.

[25] A. Schrijver, Combinatorial Optimization, Springer-Verlag, Berlin, 2003.

[26] A. Simis, W. V. Vasconcelos and R. H. Villarreal, On the ideal theory of graphs, J. Algebra 167 (1994), 389-416.

[27] R. P. Stanley, Hilbert functions of graded algebras, Adv. Math. 28 (1978), 57-83.

[28] R. P. Stanley, Decompositions of rational convex polytopes, Ann. Discrete Math. 6 (1980), 333-342.

[29] B. Sturmfels, Gröbner Bases and Convex Polytopes, University Lecture Series 8, American Mathematical Society, Rhode Island, 1996.

[30] W. V. Vasconcelos, Computational Methods in Commutative Algebra and Algebraic Geometry, Springer-Verlag, 1998.

[31] R. H. Villarreal, Monomial Algebras, Monographs and Textbooks in Pure and Applied Mathematics 238, Marcel Dekker, Inc., New York, 2001.

[32] R. H. Villarreal, Unmixed bipartite graphs, Rev. Colombiana Mat. 41(2) (2007), 393-395.

[33] R. H. Villarreal, Rees algebras and polyhedral cones of ideals of vertex covers of perfect graphs, J. Algebraic Combin. 27(3) (2008), 293-305. 